\title{
Vibrotactile frequency discrimination
}

\author{
OVE FRANZÉN \\ Department of Psychology, University of Uppsala, Uppsala, Sweden \\ and Department of Neurological Rehabilitation, Karolinska Hospital 10401 Stockholm 60, Sweden
}

and

\section{JAN NORDMARK \\ Department of Physiology II, Karolinska Institutet, 10401 Stockholm 60, Sweden}

\begin{abstract}
Thresholds for vibrotactile discrimination of pulse interval were determined for pulse frequencies between 1 and $384 \mathrm{~Hz}$. The results point to a temporal resolution significantly more accurate than that demonstrated in earlier studies. Although touch as a vibratory sensor is in general much inferior to audition, the present results show a striking resemblance to those obtained on auditory pitch. The neurophysiological implications for the tactile as well as for the auditory system are discussed.
\end{abstract}

How similar are the vibrotactile and the auditory mechanisms of frequency discrimination? The question is basic to a better understanding of both senses. Békésy (1960) has tried to show that certain inhibitory phenomena produced on the skin can serve to explain the discrepancy between the broad region of the basilar membrane set in motion by pure tones and the observed fineness of pitch discrimination.

There are obvious points of similarity between vibratory and auditory sensibility. Thresholds for vibratory sensations as a function of frequency follow the same pattern as auditory thresholds, decreasing with increasing frequency up to about the middle portion of the range of sensibility and then increasing towards higher frequencies. Furthermore, the slope of the subjective intensity power functions decreases as the carrier frequency increases to about $250 \mathrm{~Hz}$ for vibration and $1,000 \mathrm{~Hz}$ for audition (Franzén, 1969). It is less clear whether the curves for discrimination of trequency show a similar resemblance. The problem is of importance, for it bears on the question of what constitutes the code for auditory pitch. Vibratory pitch is believed to be related to the temporal pattern of impulses in the neural chain leading from the periphery to the brain. Békésy (1960), in fact, writes that the sensation of vibratory pitch is given entirely by the periodicity of the volleys in the nerves.

The present experiment tries to relate the modalities more directly by comparing the discrimination capabilities of the two senses under conditions made as closely comparable as possible. This undertaking could therefore possibly throw some light on the old controversy of place or temporal mechanisms in hearing.

Part of this investigation was supported by a grant from the Swedish Council for Social Science Research.

\section{METHOD}

Subjects

Six staff members at Karolinska Hospital took part in the experiment.

\section{Stimulation}

The fleshy pad of the middle finger was mechanically stimulated by means of a $2-\mathrm{mm}$ probe mounted on the cone of a moving-coil loudspeaker. The probe was attached to an aluminum lever projecting beyond the edge of the loudspeaker. The loudspeaker was energized by a half-wave rectitied sinusoid of $2 \mathrm{msec}$ duration. A capacitance neter connected to the probe made it possible to monitor the displacement of the rod on an oscilloscope. The capacitance variations were converted into displacement amplitudes representing the intensity of the stimulus. The test linger of the subject was kept in a tixed position by a special holder. The stimulating probe was brought into contact with the skin by means of a micromanipulator set to give a skin indentation of $5(0)$ microns. The skin-probe interface was checked by stroboscopic illumination. The standard and variable were independently generated by Wavetek generators (Model 112). The pulses were amplitied (HP power amplifier, Model 467-A) attenuated (HP attenuator set. Model $350 \mathrm{~A}$ ) to the desired level. and conducted to the tactile stimulator.

\section{Procedure}

The experimental procedure employed was almost identical to that used by Nordmark (1968) in a study on auditory pitch discrimination. The amplitude of tactile pulses perpendicularly delivered to the tinger pad was always individually set at $30 \mathrm{~dB}$ sensation level (SL) at each pulse rate. By using trains of short mechanical pulses instead of sinusoids and thus removing any ambiguity as to the time of application of the stimulus, measurenents of frequency discrimination could be extended down to $1 \mathrm{H} \%$. An added advantage to using short pulses in preterence to sinusoids was to eliminate a possible source of variation in the presentation of the vibratory stimulus. In an earlier study by Mowbray and Gebhard (1957), short pulses instead of sinusoids were used. However, as the input to the vibrating system, the authors employed rectangular pulses of varying duration and intensity. Even the shortest pulses. in this case $1.5 \mathrm{msec}$ in duration. will give rise to severe ringing. as is shown in a study by Franzên and Offenloch (1969). Figure 1 in the latter paper illustrates the response of a mechanical vibrator to a $1.5-\mathrm{msec}$ rectangular puse. The ringing is considerable even after $30 \mathrm{msec}$. 

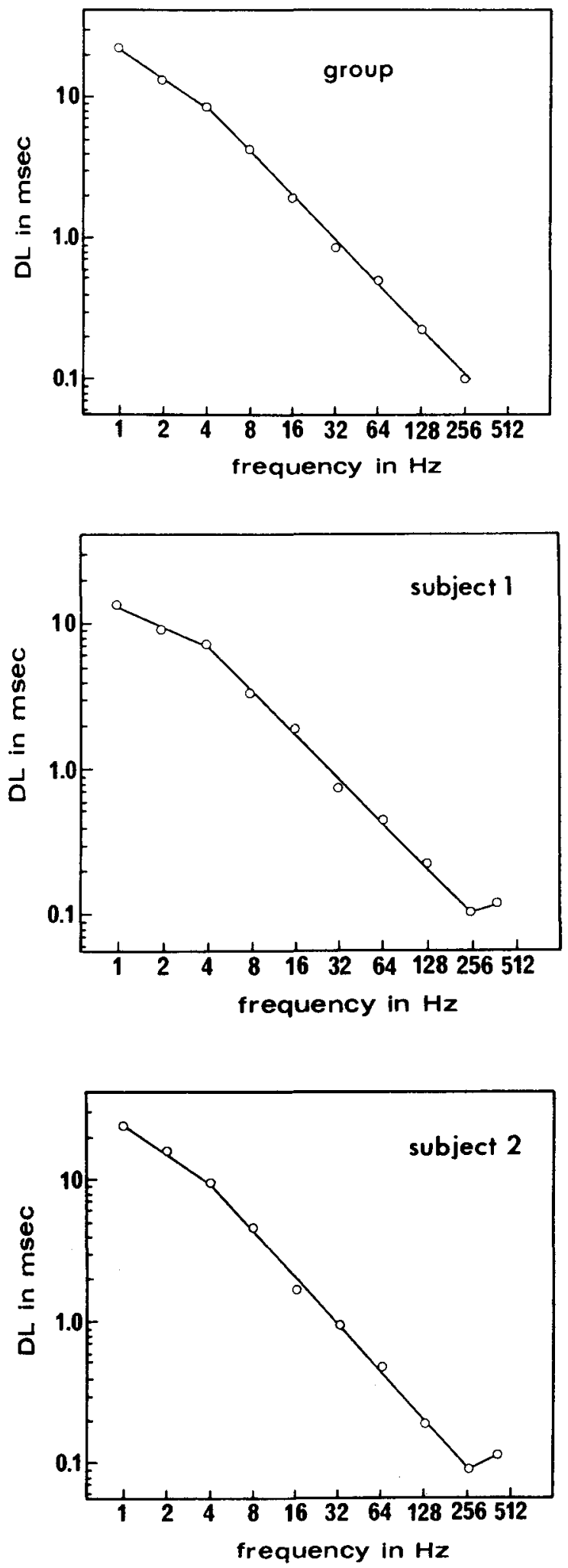

Figure 1. (a) Difference limen (DL) for short mechanical pulses as a function of frequency in hertz. Group. (b) Difference limen (DL) for short mechanical pulses as a function of frequency in hertz. Subject 1. (c) Difference limen (DL) for short mechanical pulses as a function of frequency in hertz. Subject 2.
For this reason, such pulses are unsuitable for studies on vibratory pitch discrimination for frequencies above $30-40 \mathrm{~Hz}$. The stimuli. except in the case of the lowest pulse frequencies, were a constant number of pulses during an interval of about $0.5 \mathrm{sec}$. A silent interval of 0.4 sec separated the standard stimulus from that under the subject's control. The control was effected by the main dial of a Wavetek generator in series with a 10-rotation potentiometer adjusted so that one whole rotation gave an impression of a clear change in vibratory pitch. The subject was instructed to make an approximate adjustment by means of the Wavetek generator and the fine adjustment by means of the potentiometer. He could not see the face of the Wavetek dial but was allowed to look at the potentiometer dial and, in fact, make a note of the settings arrived at by bracketing the point of equality. The time interval (period) between two pulses of the pulse train corresponding to the mean setting was then noted by the experimenter, who, after each setting changed by a random amount the dial positions of the Wavetek generator and the potentiometer. A Beckman timer determined the period. The subjects made 20 settings per frequency for rates between 1 and 256 pps. Two subjects were tested at $384 \mathrm{pps}$, which was almost the upper limit of the mechanical stimulation system. For the two highest pulse rates, the signal waveform was more nearly sinusoidal. White noise was used to mask the sound at higher frequencies.

\section{RESULTS}

The standard deviation of the settings was used as a measure of differential sensitivity (difference limen). Figure 1a gives the mean results for the subjects. They are plotted as the logarithm of the least discriminable change in time interval as a function of the logarithm of the frequency. For reasons which will be given later, the logical mode of displaying data from frequency discrimination experiments appears to be as least discriminable change in period of two different periodic stimuli rather than as change in frequency. Difference limen (DL) in Figure 1a decreases from about $10 \mathrm{msec}$ to $0.1 \mathrm{msec}$ as pulse rate is increased from 1 to $256 \mathrm{pps}$. It is interesting to note that a slight deterioration occurs at 384 pps (see Figures $1 \mathrm{~b}$ and $1 \mathrm{c})$. For comparison, data from a pitch experiment where short acoustic pulses and sinusoids have been used for stimulation are shown in Figure 2. Although touch as a vibratory sensor is, in general, much inferior to audition, the similarity between auditory and tactile frequency discrimination is striking. It is also worthy of notice, in Figures 1a, $1 \mathrm{~b}$, and 1c, that the simplest form of Weber's law holds quite well, since the slope of the function is very close to -1.0 for the major portion of the curve. The most logical way, however, of displaying data would be to plot DLs as a function of the time interval between pulses, as done in Figure 3. It is clearly seen that the uncertainty about the period is a monotonically increasing function of the time interval between pulses. The Weber fraction for DL over period was computed to be about 0.03 for almost the whole actual range (see Figure 4).

\section{DISCUSSION}

The almost constant Weber fraction for vibratory frequency discrimination was also obtained by 
Mountcastle. Talbot, Sakata, and Hyvärinen (1969). The absolute sensitivity, however, is appreciably smaller in our study. In part at least, this may be due to differences in the psychophysical method employed. A difference of similar magnitude exists between the auditory frequency discrimination ability found by Harris (1952), who used a constant method, and that found by Nordmark, who, as noted, used the same procedure as in this study.

One incidental finding in this study was that the subjects observed an experience of coherence as opposed to discreteness in the region of 4-6 Hz. This phenomenon shows up as a break in the curve (see Figure 1a). There may be analogies in other areas of perception. Thus, Johansson, Backlund, and Bergström (1969) reported an inflection point on the border between infraperceptible and perceptible motion. i.e.. between perceived change of position and motion of a target.

Our results suggest that some earlier assertions of a poor discriminative capacity of the tactile sense (Goff, 1959, 1967; Rosner, 1961) can no longer be sustained. A Weber fraction of about 0.03 is certainly very small for a system that has earlier been characterized as sluggish (Geldard, 1961).

Furthermore, two-pulse experiments do not specify appropriately the temporal acuity in somesthesis since the two successive stimuli in such experiments must be separated by at least $15 \mathrm{msec}$ in order to be perceived as separated (Rosner, 1961). This observation can be contrasted with a difference limen (DL) of 100 microsec at 256 pps.

For any given test frequency, standard and variable stimuli contained an equal number of pulses. Subjective equality was obtained by manipulation of pulse time interval. One may then ask whether duration could play any role as a subtle cue in the discrimination process, since the duration for the standard and variable stimuli was slightly different. Now, it turns out that DL for vibratory temporal discrimination is about $50 \mathrm{msec}$ (Spector, 1954) for a $500-\mathrm{msec}$ vibration, so this dimension cannot in any critical fashion influence our results.

The relation between threshold sensitivity and displacement amplitude of sinusoidal movement of the skin is best described by two functions intersecting at about 20-40 Hz (Békésy, 1960; Talbot, Darian-Smith, Kornhuber, \& Mountcastle, 1968). The dual nature of flutter vibration can readily be accounted for by the response characteristics of the quickly adapting (QA) and the Pacinian afferents innervating the hand (Talbot et al., 1968). The inflection point in the discrimination curve (see Figure 1a) cannot, however, be understood in terms of a shift from one receptor system to another.

By using short mechanical pulses instead of sinusoidal stimulation, both neural populations are in

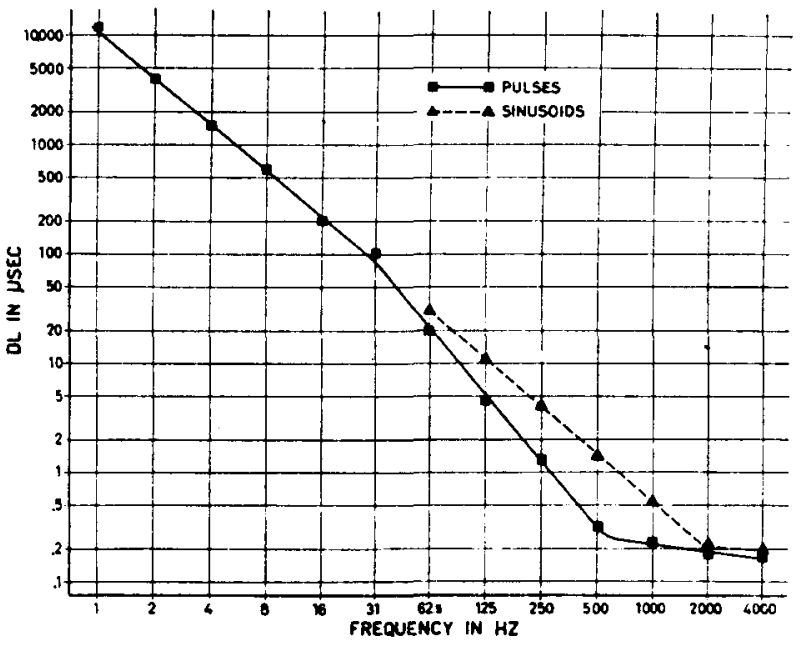

Figure 2. DLs for short acoustic pulses and sinusoids as a function of frequency. (From Nordmark, 1968).

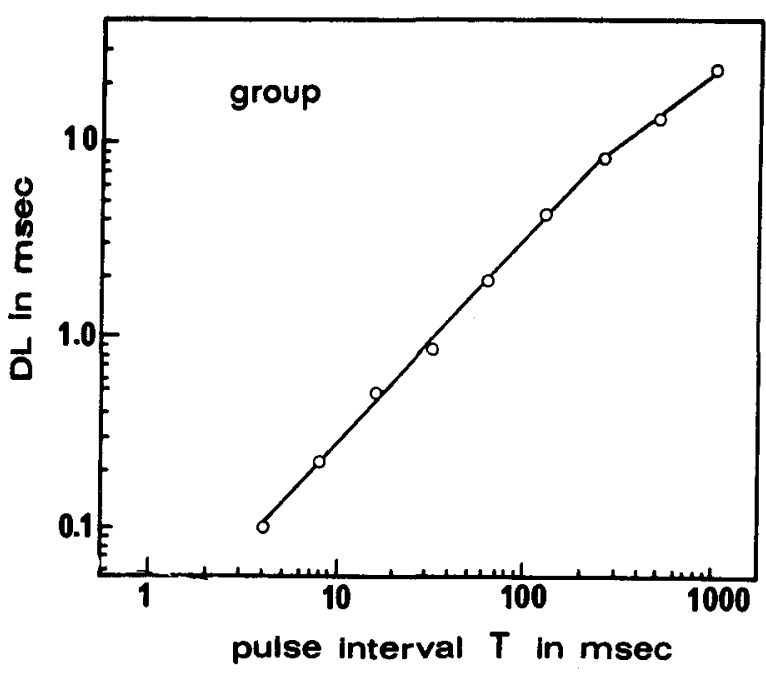

Figure 3. DL as a function of the time interval (T) between pulses. Group.

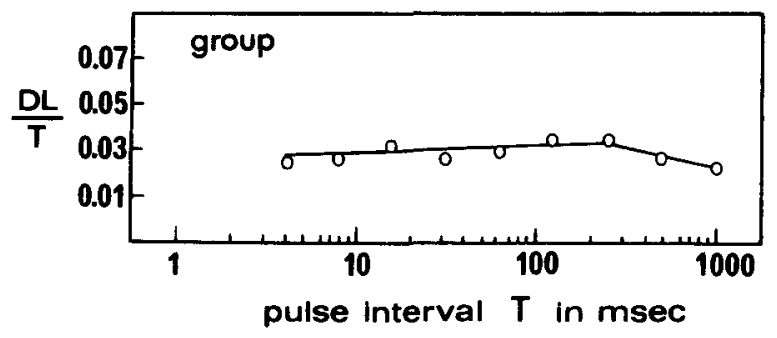

Figure 4. The Weber fraction, DL: T, as a function of the time interval between pulses. Group. 
all likelihood activated from $1 \mathrm{~Hz}$ up to the nidele range of frequencies. At higher rates, only Pacinian afferents are excited. Although one receptor system drops out, no break point is seen in the discrimination function at those rates. implying that the same underlying time mechanism is at work independently of how many or what accessible neural populations are brought into action.

Our results further accentuate a problem already raised by Mountcastle et al. (1969). Their basic assumption was that it is the periodicity in the cortical neuronal discharge which is the basis for vibratory frequency discrimination. A variability in the neuronal periods would therefore lead directly to an uncertainty in the observer's estimate of the stimulus period length. Comparing the standard deviation of the crcle histogram displaying the time of impulse discharge in cortical neurons of unanesthetized monkeys by sine-wave mechanical stimuli of various frequencies with the dispersion of estimates of period length made by humans to similar stimuli did indeed show strong resemblances. Both neuronal and psychophysical values for most frequencies dropped to stable and almost identical values as stimulus intensity rose, although the former did so at lower intensities than the latter.

Of the two classes of cortical neurons thought to play essential roles in the sense of vibration, however. the "glabrous quickly adapting" cortical neurons behaved somewhat differently from the "Pacinian" neurons. The "glabrous quickly adapting" cells. which are readily activated by stimuli in the frequency range of $5-80 \mathrm{~Hz}$, revealed a strong periodicity under low-frequency stimulation. The "Pacinian" cortical neurons are activated by high-frequency vibratory stimuli $(80-400 \mathrm{~Hz})$ delivered to peripheral tissues. just as are first-order Pacinian afferents. The periodic signals elicited in Pacinian fibers are synchronously propagated through the system. so that highfrequency periodicities appear in the discharge patterns of "Pacinian" thalamocortical fibers. But they do not occur in the discharge patterns of "Pacinian" cortical neurons. Although nonperiodic discharges appear at sine-wave amplitudes which elicit discharges in "Pacinian" thalamic afferents, the discharge rates do not differ significantly for different stimulus frequencies. Neither the overall frequency nor any temporal patterns therefore appear to provide a basis for frequency discrimination.

Because of the paradoxical results Mountcastle et al. (1969). more as a suggestion for further study than as a seriously entertained hypothesis, put forward an alternative explanation: that because of differences in mechanical coupling to the tissues, two high-frequency stimuli. e.g., 200 and $250 \mathrm{~Hz}$, will, even when equated for subjective magnitude, engage slightly different populations of cortical "Pacinian" neurons. This theory is. Mountcastle et al. (1969) note, quite comparable to the place theory of hearing.

The analogy with the place theory of hearing appears farfetched. If the discriminating cortical neuron populations correspond to differences in the patterns of stimulated Pacinian receptors, there obviously has to be a great number of identical peripheral analyzers distributed on the body, all capable of exciting the same neuron population. The number of distinguishable frequencies found by us makes the idea even less probable.

There is another, more subtle, reason for rejecting any place mechanism of vibratory pitch discrimination. Nordmark (1968) has argued that there is a fundamental difference between frequency analyzing mechanisms based on the identification of the maximally reacting part of a mechanical analyzer and that based on measurement of the period, or more generally the time interval between recurring events. While period and frequency as reciprocal quantities could theoretically be used interchangeably, frequency measurement results should clearly be displayed in terms of change in frequency in the first case and of change in time interval in the second. If. for some reason. we want to display measurements of frequency differences in terms of only one quantity, we cannot expect a continuous curve if the mechanisms involved are frequency measurements of the first kind in one frequency range and period measurement in another and partly overlapping range. For instance, the estimation of frequency for aural clicks delivered at a rate of, say, $10 / \mathrm{sec}$ is based on time interval measurement. If the pitch for a train of $1.000 / \mathrm{sec}$ clicks were based on the maximally stimulated part of the basilar membrane, lowering the click rate would give rise to a continuous curve as long as this mechanism is operative. Similarly, increasing the click rate for low-frequency clicks would give a continuous curve for the range where a time mechanism is operative. We would, however, expect a discontinuity in an overlapping frequency range if the curves were drawn only in terms of change in either frequency or period. The absence of any such discontinuity-in the mathematical sense-for auditory clicks was one reason why Nordmark concluded that pitch for periodic stimuli is based on a time-measuring mechanism.

Now, Mountcastle and his group have pointed out that they did not observe any discontinuity in the capacity for frequency discrimination as the frequency was changed from the range signaled by the glabrous quickly adapting system to that which can only depend on the Pacinian system. This fact strongly argues for periodicity as the basis for vibratory discrimination as well. How the paradox can be obviated is less clear. Perhaps the basic assumption of periodicity in the discharge of cortical neurons is unnecessary. It is at least conceivable that the periodicity that the authors found in the 
thalamocortical fibers constituting the input to the cortex. is sufficient. Since frequency discrimination can be relearned by animals with complete ablation of auditory cortex. Neff (1961) has suggested that auditory discrimination is mediated by subcortical mechanisms. The same suggestion may be applicable to the sense of touch. The cortex, being a device for processing neural concomitants of perceptual events, may not be necessary for a task requiring a decision between two frequencies.

It should, moreover, be kept in mind that a similar difficulty in reconciling the presumed temporal order in peripheral organs with measured temporal dispersion in higher centers exists also for audition (Pollack, 1968). The similarities between the two modalities emphasized in this study may perhaps be taken to indicate that the difficulties have a common explanation. There are, in any case, grounds for assuming that the perception of both vibratory and auditory pitch is based on a temporal mechanism.

\section{REFERENCES}

BÉKÉSY, G. von. Experiments in hearing. New York: McGrawHill, 1960.

FranzÉN, O. The dependence of vibrotactile threshold and magnitude functions on stimulation frequency and signal level. A perceptual and neural comparison. Scandinavian Journal of Psychology, 1969. 10. 289-298.

Franzén, O., \& OfFenloch, K. Evoked response correlates of psychophysical magnitude estimates for tactile stimulation in man. Experimental Brain Research, 1969, 8, 1-18.

Geldard, F. A. Cutaneous channels of communications. In W. A. Rosenblith (Ed.). Senson communication. New York: M.I.T. Press and Wiley, 1961. Pp. 73-87.

GoFF. G. D. Differential discrimination of frequency of cutaneous mechanical vibration. Doctoral dissertation, University of Virginia. Charlottesville, 1959.
GofF, G. D. Differential discrimination of frequency of cutaneous mechanical vibration. Joumal of Experimental Psychology. 1967, 74. 294-299.

Harrłs, J. D. Pitch discrimination. Joumal of the Acoustical Society of America, 1952, 24, 750-755.

Johansson, G., Backlund, F., \& Bergström, S. S. Shortest perceptible length of motion track as a function of stimulus velocity. In J. Järvinen (Ed.), Contemporary research in psychology of perception (In honorem Kai von Fieandt sexagenarii). Helsinki: Werner Söderström, 1969. Pp. 71-86.

Mountcastle, V. B., Talbot, W. H., Sakata, H., \& HYVÄRINEN, J. Cortical neuronal mechanisms in fluttervibration studied in unanesthetized monkeys. Joumal of Neurophy'siology, 1969, 32, 452-484.

Mowbray, G. H., \& Gebhard, J. W. Sensitivity of the skin to changes in rate of intermittent mechanical stimuli. Science. 1957, 125, 1297-1298.

NEFF, W. D. Neural mechanisms of auditory discrimination. In W. A. Rosenblith (Ed.), Sensory communication. New York: M.I.T. Press and Wiley, 1961. Pp. 259-278.

NoRDMARK. J. Mechanisnis of trequency discrimination. Journal of the Acoustical Society of America. 1968, 44, 1533-1540.

Pollack. E. Detection and relative discrimination of auditory "jitter." Journal of the Acoustical Society of America, 1968. 43. 308-315.

Rosner, B. S. Neural factors limiting cutaneous spatiotemporal discrimination. In W. A. Rosenblith (Ed.), Sensory communication. New York: M.I.T. Press and Wiley. 1961. Pp. $725-737$.

SPECTOR, P. Cutaneous communication systems utilizing mechanical vibration. Doctoral dissertation, University of Virginia. 1954.

Talbot, W. H., Darian-Smith, I.. Kornhuber, H. H., \& Mountcastle, V. B. The sense of flutter-vibration: Comparison of the human capacity with response patterns of mechanoreceptive afferents from the monkey hand. Journal of Neurophysiology. 1968, 31, 301-334.

(Received for publication May 17, 1974: revision accepted February 11, 1975.) 